\title{
The relationship between degree of facet tropism and amount of dynamic disc bulge in lumbar spine of patients symptomatic for low back pain
}

\author{
Duc H. Do - Cyrus E. Taghavi - Winston Fong • \\ Min Ho Kong • Yuichiro Morishita • \\ Jeffrey C. Wang
}

Received: 21 February 2010/Revised: 26 July 2010/ Accepted: 14 August 2010/Published online: 25 August 2010

(C) The Author(s) 2010. This article is published with open access at Springerlink.com

\begin{abstract}
Facet tropism has been investigated as a predisposing factor for degenerative changes in the lumbar spine; however, no prior study has evaluated the relationship between disc bulge and facet tropism. In this study, we used kinetic magnetic resonance imaging (kMRI) to investigate the association between degree of facet tropism and amount of disc bulge in the lumbar spine in relation to age. kMRIs in the flexion, neutral, and extension positions were performed on 410 consecutive patients with low back pain. T2-weighted midsagittal and axial mid-disc cuts were analyzed to measure disc bulge and facet angle. Facet asymmetry was calculated and classified as: no facet tropism, $<6^{\circ}$; mild facet tropism, $6-11^{\circ}$; or severe facet tropism, $\geq 11^{\circ}$. Maximal static bulge (MSB), maximal dynamic bulge (MDB), and age in the facet tropism groups were compared by age subpopulations and MDB categories, defined by the positions between which the largest change in disc bulge occurs. We found the severe facet tropism group to be associated with a nearly significant increase in MSB and MDB over the no facet tropism group in the older subpopulation at the L4-L5 level only, and a larger MDB in the L4-L5 MDB category $[E-N]$, where the greatest change in disc bulge occurs between neutral and extension positions $(p=0.013)$. Our findings suggest that severe facet tropism is associated with increased disc bulge
\end{abstract}

D. H. Do · C. E. Taghavi - W. Fong - M. H. Kong ·

Y. Morishita . J. C. Wang

Department of Orthopaedic Surgery, David Geffen School

of Medicine at UCLA, Los Angeles, CA 90095, USA

J. C. Wang $(\bowtie)$

Department of Orthopaedics and Neurosurgery, UCLA

Comprehensive Spine Center, 1250 16th Street, Suite 745,

Santa Monica, CA 90404, USA

e-mail: JWang@mednet.ucla.edu at L4-L5 in only a subset of older age patients, but may in large part be due to biomechanical factors that define the $[E-N]$ category.

Keywords Facet tropism - Lumbar disc herniation . Disc bulge $\cdot$ Kinetic magnetic resonance imaging . Disk degeneration

\section{Introduction}

Facet tropism is defined as asymmetry between the orientation angles of the right and left vertebral facet joints. In 1967, Farfan and Sullivan [1] found that tear patterns of the posterior annulus appeared to be related to facet joint asymmetry, and that there was a high association between the side of disc herniation and the more coronally facing facet joint. Thus, they hypothesized that facet tropism is associated with lumbar disc herniation (LDH). Biomechanical studies have suggested that facet tropism increases shear forces [2], making it a potential risk factor for low back pain (LBP) by predisposing individuals to degenerative diseases in the lumbar spine. Subsequent association studies have had conflicting results. The relationship between facet tropism with LDH [3-9] and facet joint osteoarthritis $[10,11]$ is contested, while no association between disc degeneration and facet tropism has been shown $[5,10,12]$.

So far, no studies have evaluated disc bulge dynamics based on the degree of facet tropism. The significance of disc bulge as a source of LBP is under continuous debate, in part due to a lack of standardized measurement criteria [13]. While there is a high prevalence in asymptomatic individuals $[14,15]$, disc bulge has been associated with annular radial tears [16] and recent sciatic pain [17]. Disc 
bulge has also been shown to increase with disc degeneration [18].

The purpose of this study was to determine if the degree of facet tropism was associated with differences in disc bulge dynamics at the L3-L4, L4-L5, and L5-S1 levels in symptomatic patients, and how age affects this association by using kinetic magnetic resonance imaging (kMRI).

\section{Materials and methods}

\section{Study population}

Between November 2004 and July 2008, 410 consecutive patients symptomatic for LBP were referred for MR analysis by kMRI. Patients with spondylolisthesis, spinal infection, severe scoliosis or a history of previous spinal surgery were excluded from this study. There were 270 males and 140 females with a mean age of 40.7 years (range 16-80 years).

\section{Imaging instrumentation}

MRI of the lumbar spine was performed using a 0.6 T MRI scanner (UPRIGHT ${ }^{\mathrm{TM}}$ Multi-Position, Fonar Corporation, New York, NY). The MR unit uses two vertically oriented, opposing magnet doughnuts, allowing for scanning of the patient in an upright weight-bearing position. Images were obtained using a quad channel planar coil. T2-weighted images were taken by fast spin echo (repetition time $3,000 \mathrm{~ms}$, echo time $140 \mathrm{~ms}$, thickness $4.0 \mathrm{~mm}$, field of view $30 \mathrm{~cm}$, matrix $256 \times 224$, NEX, 2, flip angle).

\section{Procedure}

Each patient was imaged in the weight-bearing neutral, flexion, and extension positions. Points were marked by spine surgeons on each T2-weighted midsagittal cut containing the L3-L4, L4-L5, and L5-S1 discs for digitization. At each level, four points were marked for each vertebral body (anterior-superior, anterior-inferior, posterior-superior, and posterior-inferior), two points for disc height at the middle of each endplate, and two points for spinal canal diameter and pedicle diameter (Fig. 1). Disc bulge was measured from these marked films using MRI Analyzer Version 3 (Truemetric Corporation, Bellflower, California) for objective analysis and recorded in millimeters.

Facet angle (FA) was measured from T2-weighted axial cuts at the mid-disc level. Spine surgeons drew facet lines connecting the margins of the superior articular facet as well as a reference midsagittal line, and the FA between those lines was calculated bilaterally using MRI Analyzer

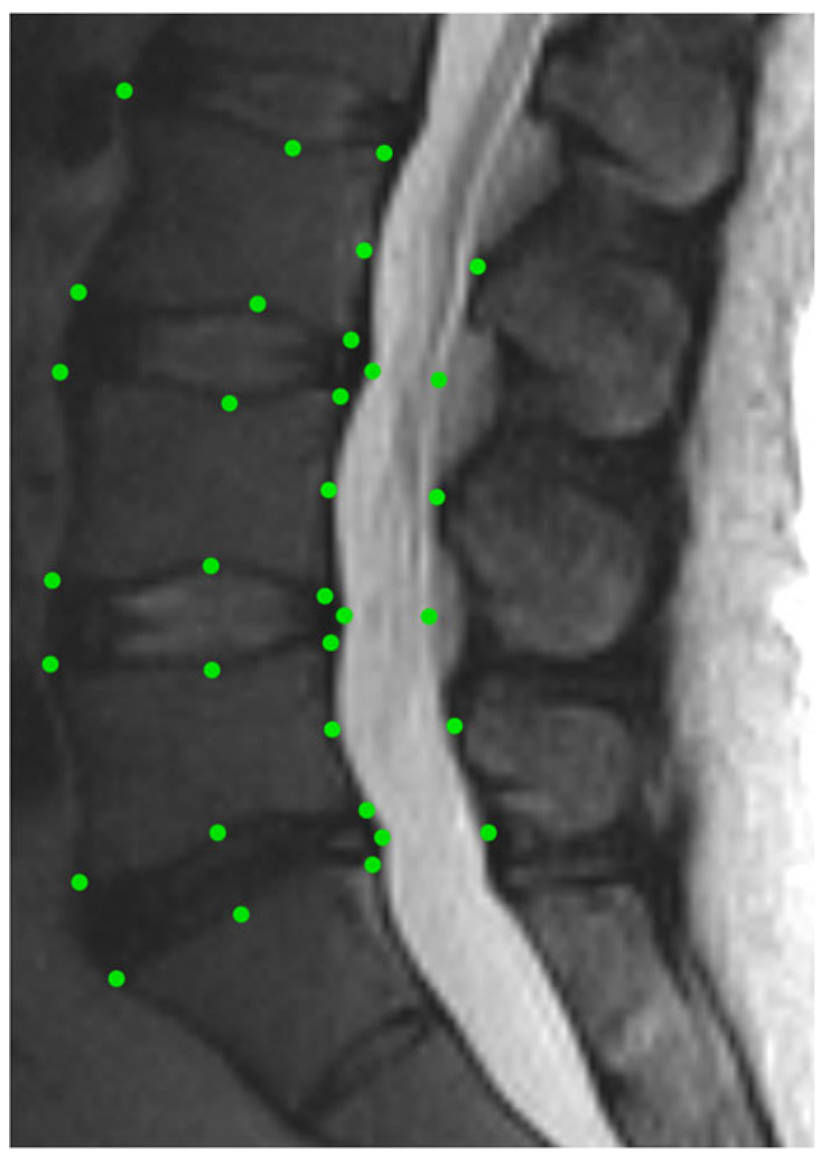

Fig. 1 Disc bulge measurement. Using T2-weighted midsagittal cuts from L3-S1, points were marked at the corner of each vertebral body, at the midpoint of the endplates, for spinal canal diameter and pedicle height. Disc bulge was calculated using this marked image by MRI Analyzer Version 3 (Truemetric Corporation, Bellflower, CA)

Version 3 (Fig. 2). This was repeated for each of the L3L4, L4-L5, and L5-S1 facet joints.

This method of measurement has been shown previously by our group to have good intraobserver and interobserver reliability [18].

\section{Evaluation}

Facet asymmetry was calculated at each level as the positive difference between $\mathrm{FA}_{\text {right }}$ and $\mathrm{FA}_{\text {left }}$. The facet joints were classified into facet tropism groups based on the degree of facet asymmetry: FT - (no facet tropism $<6^{\circ}$ ), $\mathrm{FT}+\left(\right.$ mild facet tropism $6-11^{\circ}$ ), and FT ++ (severe facet tropism $\geq 11^{\circ}$ ). The categories were determined by using the distribution of facet asymmetry at the L4-L5 level where $6^{\circ}$ is the mean and $11^{\circ}$ is +1 standard deviation (SD, Table 1).

Two disc bulge measurements were used: maximal static bulge (MSB) and maximal dynamic bulge (MDB). MSB was calculated by taking the largest disc bulge value 
Fig. 2 Facet angle measurement. A facet line connecting the margins of the superior facet margin was drawn for each facet joint, and the angle with respect to the reference midsagittal line $\left(\alpha_{\mathrm{L}}\right.$ and $\left.\alpha_{\mathrm{R}}\right)$ was calculated by MRI Analyzer 3 bilaterally

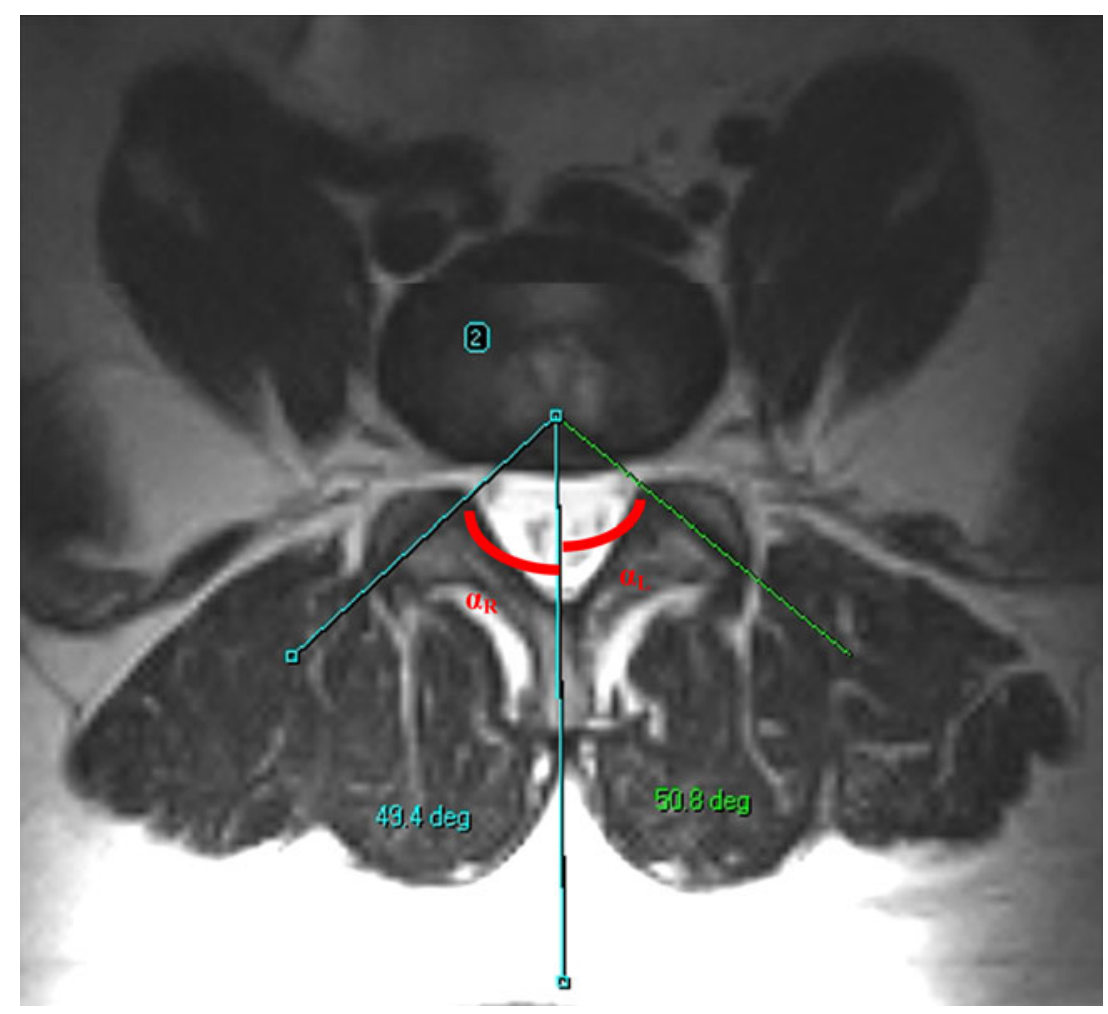

at a level for a patient among the neutral $(N)$, flexion $(F)$, or extension $(E)$ positions. MDB was defined as the largest change in disc bulge between any of the three positions (i.e., the largest of the three values obtained from the calculations $|F-N|,|E-F|$, and $|E-N|)$.

\section{Analysis}

Comparisons of age, MSB, and MDB between the facet tropism groups were made independently at each disc level in the overall population, the subpopulation with age $<41$ years (mean age), and the subpopulation with age $\geq 41$ years by ANOVA. Three MDB categories $([F-N]$, $[E-F]$, and $[E-N])$ were also considered based on the positions between which the largest change in disc bulge was observed (e.g., disc levels at which the largest change in disc bulge is between flexion and neutral positions were placed in the $[F-N]$ category). Similar comparisons were made within each of these categories in the overall population and both age subpopulations. Comparisons between MDB categories were performed by ANOVA. Tukey's test was used to determine pairwise significance. Comparisons between age subpopulations were performed by Student's $t$ test. All statistical analyses were done using SPSS software (Version 16, Chicago, IL) and a $p$ value equal to or less than 0.05 was considered to be statistically significant.

\section{Results}

The distribution of facet asymmetry had a mean of $5.9 \pm 5.1^{\circ}$ at $\mathrm{L} 3-\mathrm{L} 4,6.0 \pm 5.0^{\circ}$ at $\mathrm{L} 4-\mathrm{L} 5$, and $6.3 \pm 5.5^{\circ}$ at L5-S1, which were not significantly different $(p=$ 0.537). Mild facet tropism was found in $114(28 \%), 113$ (28\%), and 100 (24\%) patients at the L3-L4, L4-L5, and L5-S1 levels, respectively. Severe facet tropism was found in $56(14 \%), 63(15 \%)$, and $70(17 \%)$ of the patients at the L3-L4, L4-L5, and L5-S1 levels, respectively. Mean disc bulge by positions is shown in Table 2 .

Relationship between MSB/MDB and age

MSB increased significantly with age at both L3-L4 and L4-L5, while MDB did not increase with age at any level (Table 3). MSB correlated significantly with MDB at L3-L4 (Pearson $r=0.487, p<0.001$ ), L4-L5 (Pearson $r=0.491, p<0.001$ ), and L5-S1 (Pearson $r=0.526$, $p<0.001)$.

\section{Comparison between facet tropism groups}

In the overall population, there was a trend for disc bulge to increase with higher degree of facet tropism at L4-L5, but there was no significant difference in either MSB $(p=0.275)$ or MDB ( $p=0.167$, Fig. 3$)$. A nonsignificant 
Table 1 Demographics of facet tropism groups by age

\begin{tabular}{|c|c|c|c|c|}
\hline \multirow[t]{2}{*}{ Age } & \multicolumn{3}{|c|}{ Degree of facet tropism } & \multirow[t]{2}{*}{$p$} \\
\hline & $\mathrm{FT}-$ & FT + & $\mathrm{FT}++$ & \\
\hline \multicolumn{5}{|l|}{$<41$} \\
\hline \multicolumn{5}{|l|}{ L3-L4 } \\
\hline$n$ & $119(58)$ & $57(28)$ & $28(14)$ & \\
\hline Age (years) & $31.1 \pm 5.9$ & $31.4 \pm 5.9$ & $32.8 \pm 6.0$ & 0.383 \\
\hline Male & $79(66)$ & $41(72)$ & $25(89)$ & 0.055 \\
\hline \multicolumn{5}{|l|}{ L4-L5 } \\
\hline$n$ & $123(60)$ & $54(27)$ & $27(13)$ & \\
\hline Age (years) & $31.6 \pm 5.9$ & $31.4 \pm 5.9$ & $30.5 \pm 6.1$ & 0.653 \\
\hline Male & $86(70)$ & $40(74)$ & $19(70)$ & 0.851 \\
\hline \multicolumn{5}{|l|}{ L5-S1 } \\
\hline$n$ & $123(60)$ & $48(24)$ & $33(16)$ & \\
\hline Age (years) & $31.5 \pm 5.9$ & $31.4 \pm 6.3$ & $31.2 \pm 5.7$ & 0.964 \\
\hline Male & 85 (69) & $32(67)$ & $28(85)$ & 0.155 \\
\hline \multicolumn{5}{|l|}{$\geq 41$} \\
\hline \multicolumn{5}{|l|}{ L3-L4 } \\
\hline$n$ & $121(59)$ & $57(28)$ & $28(14)$ & \\
\hline Age (years) & $49.0 \pm 6.7$ & $50.8 \pm 7.4$ & $51.4 \pm 9.3$ & 0.143 \\
\hline Male & $71(59)$ & $36(63)$ & $18(64)$ & 0.778 \\
\hline \multicolumn{5}{|l|}{ L4-L5 } \\
\hline$n$ & $111(54)$ & $59(29)$ & $36(17)$ & \\
\hline Age (years) & $48.4 \pm 6.3$ & $50.6 \pm 7.7$ & $52.9 \pm 8.8$ & 0.004 \\
\hline Male & $64(58)$ & $37(63)$ & $24(67)$ & 0.586 \\
\hline \multicolumn{5}{|l|}{ L5-S1 } \\
\hline$n$ & 117 (57) & $52(25)$ & 37 (18) & \\
\hline Age (years) & $49.4 \pm 7.0$ & $49.0 \pm 6.2$ & $52.4 \pm 9.2$ & 0.056 \\
\hline Male & 68 (58) & $30(58)$ & $27(73)$ & 0.239 \\
\hline
\end{tabular}

Values in parentheses are percentages. Values for age are mean \pm SD $F T$ - no facet tropism, $F T+$ mild facet tropism, $F T++$ severe facet tropism

Table 2 Disc bulge by position

\begin{tabular}{lllll}
\hline & \multicolumn{2}{l}{ Position } & \multirow{2}{*}{$p$} \\
\cline { 2 - 4 } & Neutral $(\mathrm{mm})$ & Flexion $(\mathrm{mm})$ & Extension $(\mathrm{mm})$ & \\
\hline L3-L4 & $2.90 \pm 1.31$ & $2.73 \pm 1.37^{*}$ & $3.04 \pm 1.44^{*}$ & 0.005 \\
L4-L5 & $3.61 \pm 1.60$ & $3.45 \pm 1.61^{*}$ & $3.79 \pm 1.58^{*}$ & 0.008 \\
L5-S1 & $3.57 \pm 1.78$ & $3.49 \pm 1.73$ & $3.71 \pm 1.87$ & 0.215 \\
\hline
\end{tabular}

Values are mean $\pm \mathrm{SD}$

$* p<0.05$ pair-wise comparison

trend for age to increase with facet asymmetry was found at L4-L5 $(p=0.052)$. No significant differences were found at L3-L4 and L5-S1.

Comparison of MSB and MDB between the age $<41$ and age $\geq 41$ subpopulations revealed a significant difference in $\mathrm{MDB}$ in the $\mathrm{FT}++$ group between the two subpopulations at L4-L5 ( $p=0.005$, Fig. 4). Within the subpopulation with age $<41$ years, there were no significant differences between the facet tropism groups at any level.

In the subpopulation with age $\geq 41$ years at L4-L5, there was a significant difference in MSB $(p=0.032)$ between the facet tropism groups and a nonsignificant trend for MDB to increase with facet asymmetry $(p=0.065$, Fig. 4). There was a trend for increasing age with increasing severity of facet tropism at all levels, but only reaching significance at L4-L5 $(p=0.004$, Table 1). No significant differences were found at L3-L4 or L5-S1.

Comparison between facet tropism groups within MDB categories

Mean MSB and MDB in the MDB categories are shown in Table 4. In the FT++ group at L4-L5, mean MDB in the $[E-N]$ category was significantly higher than that of the other two categories $(p=0.049$, Fig. 5). Comparisons between facet tropism groups within the MDB categories revealed a significant difference in age only in the $[E-N]$ category at L3-L4 $(p=0.020)$ and L4-L5 $(p=0.011$, Table 5). At L4-L5, we found a significant difference in MDB between the facet tropism groups in the $[E-N]$ category $(p=0.018$, Fig. 5). No differences reached significance in the $[F-N]$ and $[E-F]$ categories at any level.

No significant differences were found at any level within the MDB categories when only the subpopulation with age $<41$ years was considered. Similar comparisons considering only the subpopulation with age $\geq 41$ years found significant differences in age between the facet tropism groups in the $[E-N]$ category, at L3-L4 $(p=0.028)$ and L4-L5 $(p=0.013$, Table 5). No significant differences in MSB were found in any MDB category. There was a significant difference in MDB in the $[E-N]$ category only $(p=0.049)$ at L4-L5, particularly between FT $++(2.03 \pm 1.12)$ and FT $-(1.32 \pm 0.68$, Fig. 6$)$. There were no significant differences at the L5-S1 level, although a similar trend in age was found in the $[E-N]$ category $(p=0.228)$.

\section{Discussion}

Facet tropism has been defined using various methods. Cyron and Hutton [2] defined it as bilateral angle differences of greater than $1^{\circ}$. Noren et al. [7] used $5^{\circ}$ as a cutoff. Ko and Park [3] defined it as asymmetry greater than $1 \mathrm{SD}$ at that level. Boden et al. used one definition for all levels based on percentiles of asymmetry in asymptomatic volunteers [4]. In this study, we defined mild facet tropism as a bilateral angle positive difference between the mean $\left(6^{\circ}\right)$ 
Table 3 MSB and MDB by age group

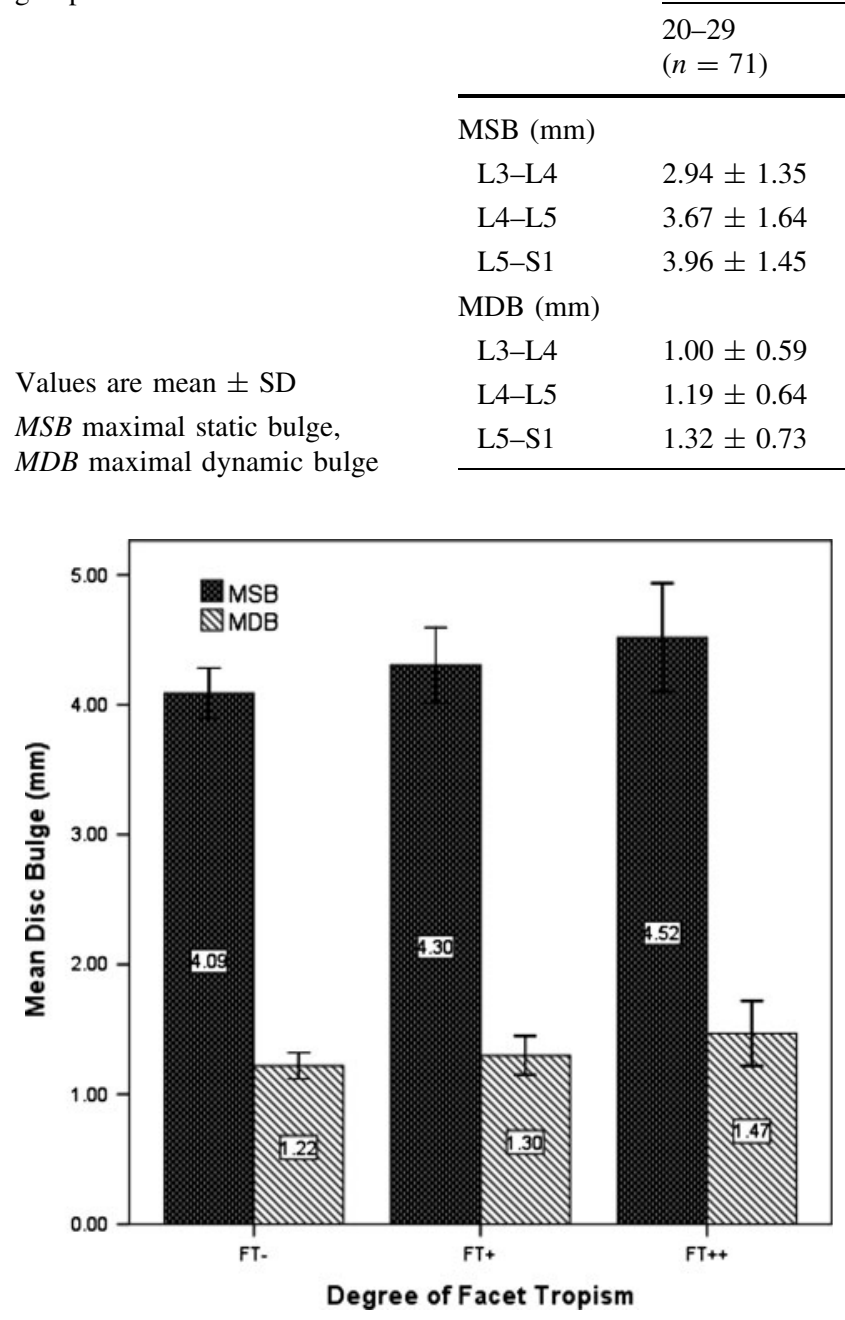

Fig. 3 L4-L5: MSB, and MDB in the overall population. There was no significant difference in either MSB or MDB between the facet tropism groups

and +1 SD $\left(11^{\circ}\right)$, based on the L4-L5 distribution, and severe facet tropism as a difference greater than $+1 \mathrm{SD}$. Although mean facet asymmetry increases from L3-L4 to L5-S1, the distribution of facet asymmetry at those levels were not significantly different; hence, we used a single definition for all three levels. We also observed that age was associated with increased severity of facet tropism, especially in the $[E-N]$ category, an observation also made by Kalichman et al. [11].

The significance of disc bulge and its relationship to LBP is under debate, as there is a high prevalence of disc bulge in reportedly asymptomatic patients $[14,15]$. In a study relating radiographic imaging and discographic findings of cadaveric lumbar discs, Yu et al. [16] found that almost all discs with no radial tears had less than $2.5 \mathrm{~mm}$ disc-bulging index, while $84 \%$ of discs with radial tears had disc-bulging index greater than $2.5 \mathrm{~mm}$. Thus, they suggested an association between disc bulge and annular tears, which can be a source of LBP due to their anatomical relationship with a richly innervated area of the disc [19]. Milette et al. [20] reported similar findings and added that disc bulges and protrusions represent discs with similar internal architecture. Stadnik et al. [19], however, did not find a significant association. Buirski et al. [21] also suggested that disc bulge offers little predictive value in addition to other MRI characteristics of disc degeneration to predict pain reproduced on discography, a finding shared by Milette et al. [20]. Comparison of findings from studies involving disc bulge, however, is difficult due to lack of common measurement criteria [13]. In addition, since the measurements used by $\mathrm{Yu}$ et al. were from a recumbent position, it is not possible to compare them with our findings from the axially loaded position that is generally greater, even in the neutral position [22, 23].

Luoma et al. [17] reported a significant association between self-reported sciatic pain but not non-sciatic LBP in the past 12 months with posterior disc bulge on MRI, reported as disc bulge measurements over $3.2 \mathrm{~mm}$. Jensen et al. [24] showed that while $22 \%$ of disc bulge were associated with sciatica, only $6 \%$ were associated with nerve root compression. In a recent kMRI study, Zou et al. [25] showed that extension and flexion in the axially loaded position could reveal clinically significant disc bulges that are read as benign on conventional MRI. In another kMRI study, Zou et al. [18] showed that disc bulge increases with disc degeneration. This study also demonstrated that with increasing disc degeneration, posterior disc bulges may increase significantly with extension, a finding supported by other studies [23]. This suggests that with increasing disc degeneration, the possibility of disc bulge causing positional sciatic pain, especially in extension, increases. However, the extent of disc bulge required to cause nerve root compression is still unclear.

In this study, we introduced two measurements, MSB and MDB. MSB is the largest disc bulge measurement for a 


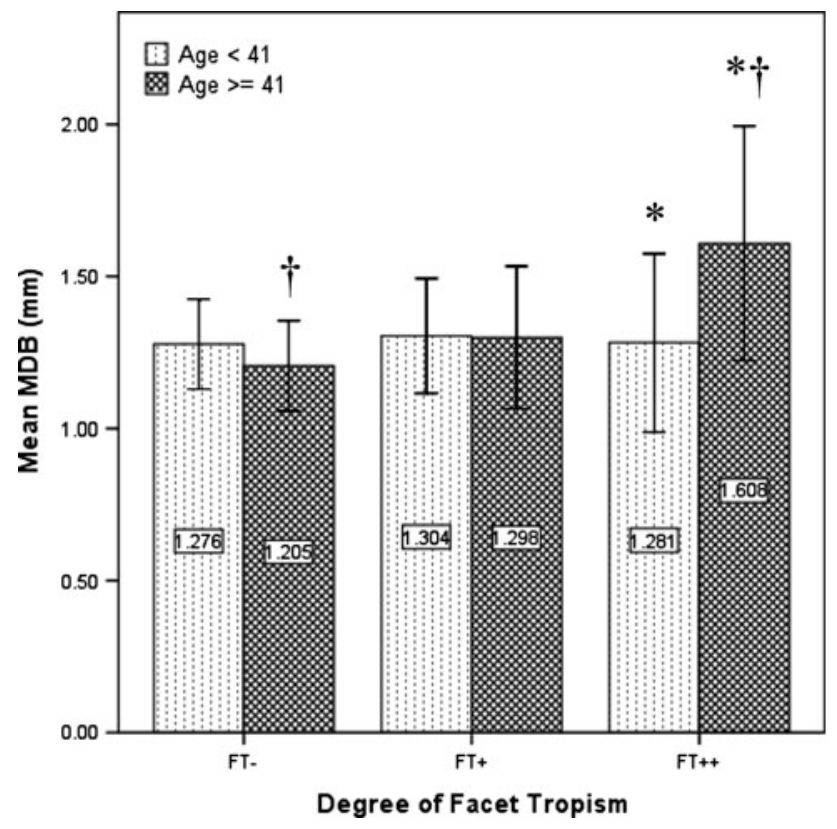

Fig. 4 L4-L5: MDB between age subpopulations and facet tropism groups. There is a significant difference in MDB between the age subpopulations in the $\mathrm{FT}++$ group only. There is a significant difference in $\mathrm{MDB}$ between $\mathrm{FT}++$ and $\mathrm{FT}-$ groups in the subpopulation with age $\geq 41$ years only $\left({ }^{*} p<0.01,{ }^{\dagger} p=0.051\right)$

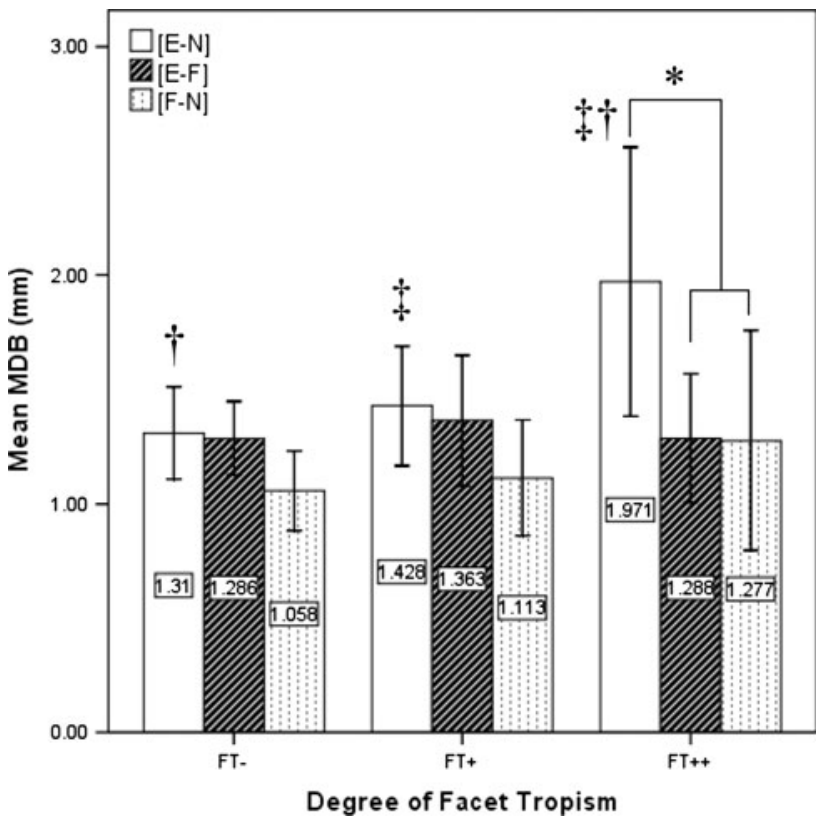

Fig. 5 L4-L5: MDB in MDB categories and facet tropism groups. There is a significant difference in MDB between the MDB categories in the FT ++ group only. There is a significant difference in MDB between $\mathrm{FT}++$ and $\mathrm{FT}-$, and trend toward difference between $\mathrm{FT}++$ and FT $+\left({ }^{*} p<0.05,{ }^{\dagger} p<0.05,{ }^{\ddagger} p=0.076\right)$
Table 4 Characteristics of maximal dynamic bulge categories

\begin{tabular}{|c|c|c|c|c|c|}
\hline & \multirow[t]{2}{*}{ Overall } & \multicolumn{3}{|c|}{ Maximal dynamic bulge category } & \multirow[t]{2}{*}{$p$} \\
\hline & & {$[F-N]$} & {$[E-F]$} & {$[E-N]$} & \\
\hline \multicolumn{6}{|l|}{ L3-L4 } \\
\hline$n$ & 410 & $150(37)$ & $159(39)$ & $101(25)$ & \\
\hline Age (years) & $40.7 \pm 11.4$ & $40.2 \pm 10.9$ & $40.7 \pm 11.0$ & $41.3 \pm 12.6$ & 0.747 \\
\hline Male & $270(66)$ & $101(67)$ & $104(66)$ & $65(64)$ & 0.878 \\
\hline $\operatorname{MSB}(\mathrm{mm})$ & $3.4 \pm 1.4$ & $3.1 \pm 1.2^{* *}$ & $3.5 \pm 1.4^{*}$ & $3.7 \pm 1.7^{*}$ & 0.002 \\
\hline $\operatorname{MDB}(\mathrm{mm})$ & $1.1 \pm 0.7$ & $0.9 \pm 0.6^{* *}$ & $1.2 \pm 0.8^{*}$ & $1.1 \pm 0.7^{*}$ & 0.001 \\
\hline \multicolumn{6}{|l|}{ L4-L5 } \\
\hline$n$ & 410 & $136(33)$ & $158(39)$ & $116(28)$ & \\
\hline Age (years) & $40.7 \pm 11.4$ & $39.1 \pm 11.2^{*}$ & $42.4 \pm 11.4 *$ & $41.1 \pm 11.3$ & 0.036 \\
\hline Male & $270(66)$ & $82(60)$ & $108(68)$ & $80(69)$ & 0.246 \\
\hline $\operatorname{MSB}(\mathrm{mm})$ & $4.3 \pm 1.6$ & $3.9 \pm 1.7 *^{\dagger}$ & $4.4 \pm 1.6^{*}$ & $4.5 \pm 1.6^{\dagger}$ & 0.007 \\
\hline $\operatorname{MDB}(\mathrm{mm})$ & $1.3 \pm 0.8$ & $1.1 \pm 0.8^{\ddagger}$ & $1.3 \pm 0.8$ & $1.4 \pm 0.9^{*}$ & 0.010 \\
\hline \multicolumn{6}{|l|}{ L5-S1 } \\
\hline$n$ & 410 & $144(35)$ & $129(32)$ & $137(33)$ & \\
\hline Age (years) & $40.7 \pm 11.4$ & $40.5 \pm 11.4$ & $40.3 \pm 10.8$ & $41.4 \pm 11.9$ & 0.828 \\
\hline Male & $270(66)$ & $93(65)$ & $82(64)$ & $95(69)$ & 0.564 \\
\hline MSB (mm) & $4.3 \pm 1.8$ & $4.2 \pm 1.7$ & $4.1 \pm 1.8$ & $4.6 \pm 1.9$ & 0.121 \\
\hline MDB (mm) & $1.4 \pm 1.0$ & $1.3 \pm 1.1$ & $1.5 \pm 0.9$ & $1.5 \pm 1.0$ & 0.169 \\
\hline
\end{tabular}

level from the neutral, flexion, and extension positions. Since a spinal motion segment is dynamic, MSB allows us to assess for nerve impingement potential more adequately than measurements from any single position. However,
MSB may be of limited usefulness for comparison across large age ranges, because it increases with age at all levels, likely due to disc degeneration, a finding supported by other studies [15, 18]. MDB, however, defined as the 
Table 5 Age versus severity of facet tropism in maximal dynamic bulge categories

\begin{tabular}{ccccc}
\hline & \multicolumn{2}{l}{ Degree of facet tropism } & $p$ \\
\cline { 2 - 4 } & FT- (years) & FT + (years) & FT++ (years) & \\
\hline L3-L4 & & & & \\
Overall & $40.1 \pm 10.9$ & $41.1 \pm 11.8$ & $42.1 \pm 12.2$ & 0.400 \\
{$[E-N]$} & $39.2 \pm 10.6^{*}$ & $41.6 \pm 13.9$ & $50.7 \pm 15.0^{*}$ & 0.020 \\
{$[E-F]$} & $40.5 \pm 10.9$ & $42.9 \pm 11.4$ & $36.9 \pm 9.3$ & 0.173 \\
{$[F-N]$} & $40.2 \pm 11.3$ & $38.9 \pm 10.2$ & $41.7 \pm 11.0$ & 0.571 \\
L4-L5 & & & & \\
Overall & $39.6 \pm 10.4$ & $41.4 \pm 11.8$ & $43.3 \pm 13.6$ & 0.052 \\
{$[E-N]$} & $38.0 \pm 10.0^{*}$ & $40.6 \pm 11.4$ & $47.1 \pm 12.7 *$ & 0.011 \\
{$[E-F]$} & $41.4 \pm 10.2$ & $44.5 \pm 12.2$ & $43.3 \pm 14.4$ & 0.337 \\
{$[F-N]$} & $38.7 \pm 10.6$ & $39.1 \pm 11.3$ & $40.4 \pm 13.2$ & 0.826 \\
L5-S1 & & & & \\
Overall & $40.2 \pm 11.0$ & $40.5 \pm 10.8$ & $42.4 \pm 13.2$ & 0.358 \\
{$[E-N]$} & $40.2 \pm 11.5$ & $41.4 \pm 12.4$ & $44.2 \pm 12.7$ & 0.328 \\
{$[E-F]$} & $40.4 \pm 10.7$ & $39.8 \pm 10.5$ & $40.7 \pm 12.2$ & 0.954 \\
{$[F-N]$} & $40.1 \pm 11.0$ & $40.6 \pm 10.1$ & $42.2 \pm 15.1$ & 0.741 \\
\hline
\end{tabular}

Values are mean $\pm \mathrm{SD}$

$F T-$ no facet tropism, $F T+$ mild facet tropism, $F T++$ severe facet tropism

$* p<0.05$ pair-wise comparison

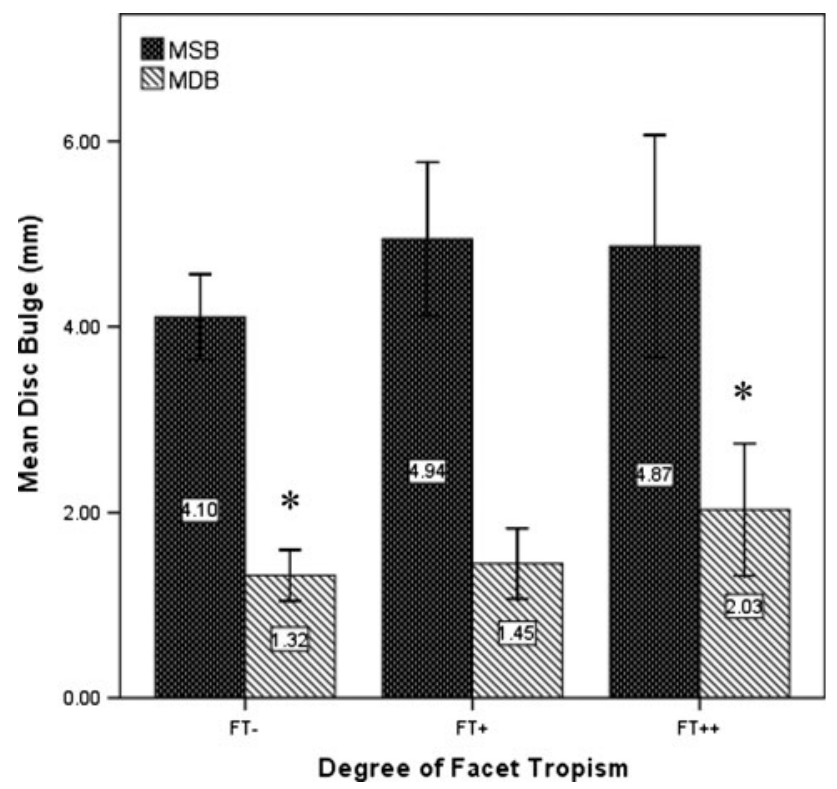

Fig. 6 L4-L5: MSB and MDB in $[E-N]$ category, age $\geq 41$ years. There was a significant difference in MDB between FT ++ and FT groups $(* p<0.05)$

difference between the maximal and minimal static disc bulge measurements, does not increase with age but still correlates significantly with MSB at all three levels. Since this study includes a large range of ages (16-80 years),
MDB allows us to study the dynamics of disc bulge while controlling for age-related changes.

While studies investigating associations between facet tropism and degenerative changes are controversial, there is general agreement that any association only occurs at L4-L5, due to its greater mobility and inherent instability $[4,5,10]$. We present similar findings. We further show that only the combination of both severe facet tropism and older age is associated with increased disc bulge at L4-L5, highlighting the importance of considering the role age plays in the relationship between facet tropism and degenerative changes.

We further characterized patients by MDB category, based on the positions that yielded the largest change in disc bulge. We show that at L3-L4 and L4-L5, only the group with the largest change in disc bulge between extension and neutral positions (the $[E-N]$ category) is significantly correlated with increasing severity of facet tropism in older age. In addition, only in this category of patients is severe facet tropism at the L4-L5 level associated with increased MDB in old age. It is not completely clear what causes the largest change in disc bulge to occur between certain positions. Past studies have shown consistently that in non-degenerated discs, posterior disc surface migrates posteriorly with flexion and anteriorly with extension [18]. In more degenerate discs, posterior disc surface migration is more unpredictable, but at L3-L4 and L4-L5, extension generally causes an increase in disc bulge measurements [18]. Hence, degenerative changes may be partially responsible for classifying a disc in the $[E-N]$ category at L3-L4 and L4-L5, but given the variability there are likely other, yet to be elucidated biomechanical factors responsible.

Our findings, however, suggest that at L4-L5, degenerated discs in older patients that fit in this category and occur in the presence of severe facet tropism may have similar disc bulge measurements in the neutral position as those occurring without facet tropism, but have significantly increased disc bulge in extension that may contribute to sciatic pain. Hence, in older patients who have sciatic pain correlating to L4-L5 disc degeneration and severe facet tropism, but no apparent source of pain on conventional MRI, it may be worthwhile to order kMRI to evaluate for nerve compression due to posterior disc bulging upon extension in the axially loaded position.

A limitation of this study is the lack of asymptomatic controls. To our knowledge, there are currently no studies that measure the extent of disc bulge in asymptomatic patients. We also did not assess for signs of nerve compression in this study.

Open Access This article is distributed under the terms of the Creative Commons Attribution Noncommercial License which permits any noncommercial use, distribution, and reproduction in any medium, provided the original author(s) and source are credited. 


\section{References}

1. Farfan H, Sullivan J (1967) The relation of facet orientation to intervertebral disc failure. Can J Surg 10:179-185

2. Cyron B, Hutton W (1980) Articular tropism and stability of the lumbar spine. Spine 5:168-172

3. Ko H, Park B (1997) Facet tropism in lumbar motion segments and its significance in disc herniation. Arch Phys Med Rehabil 78:1211-1214

4. Hägg O, Wallner A (1990) Facet joint asymmetry and protrusion of the intervertebral disc. Spine 15:356-359

5. Boden S, Riew K, Yamaguchi K, Branch TP, Schellinger D, Wiesel SW (1996) Orientation of the lumbar facet joints: association with degenerative disc disease. J Bone Jt Surg Am 78:403-411

6. Kunakornsawat S, Ngamlamaidt K, Tungsiripat R, Prasartritha T (2007) The relationship of facet tropism to lumbar disc herniation. J Med Assoc Thail 90:1337-1341

7. Noren R, Trafimow J, Andersson G, Huckman MS (1991) The role of facet joint tropism and facet angle in disc degeneration. Spine 16:530-532

8. Van Schaik J, Verbiest H, Van Schaik F (1985) The orientation of laminae and facet joints in the lower lumbar spine. Spine 10:59-63

9. Karacan I, Aydin T, Sahin Z, Cidem M, Koyuncu H, Aktas I, Uludag M (2004) Facet angles in lumbar disc herniation: their relation to anthropometric features. Spine 29:1132-1136

10. Kong MH, He W, Tsai YD, Chen NF, Keorochana G, Do DH, Wang JC (2009) Relationship of facet tropism with degeneration and stability of functional spine unit. Yonsei Med J 50:624-629

11. Kalichman L, Suri P, Guermazi A, Li L, Hunter DJ (2009) Facet orientation and tropism: associations with facet joint osteoarthritis and degeneratives. Spine 34:E579-E585

12. Vanharanta H, Floyd T, Ohnmeiss D, Hochschuler SH, Guyer RD (1993) The relationship of facet tropism to degenerative disc disease. Spine 18:1000-1005

13. Brant-Zawadzki MN, Jensen MC, Obuchowski N, Ross JS, Modic MT (1995) Interobserver and intraobserver variability in interpretation of lumbar disc abnormalities: a comparison of two nomenclatures. Spine 20:1257-1264

14. Jarvik JG, Deyo RA (2002) Diagnostic evaluation of low back pain with emphasis on imaging. Ann Intern Med 137:586-597
15. Jensen MC, Brant-Zawadzki MN, Obuchowski N, Modic MT, Malkasian D, Ross JS (1994) Magnetic resonance imaging of the lumbar spine in people without back pain. $\mathrm{N}$ Engl J Med 331:69-73

16. Yu S, Haughton VM, Sether LA, Wagner M (1988) Annulus fibrosus in bulging intervertebral disks. Radiology 169:761-763

17. Luoma K, Riihimaki H, Luukkonen R, Raininko R, ViikariJuntura E, Lamminen A (2000) Low back pain in relation to lumbar disc degeneration. Spine 25:487-492

18. Zou J, Yang H, Miyazaki M, Morishita J, Wei F, McGovern S, Wang JC (2009) Dynamic bulging of intervertebral discs in the degenerative lumbar spine. Spine 34:2545-2550

19. Stadnik TW, Lee RR, Coen HL, Neirynck EC, Buisseret TS, Oxteaux MJC (1998) Annular tears and disk herniation: prevalence and contrast enhancement on MR images in the absence of low back pain or sciatica. Radiology 206:49-55

20. Milette PC, Suzanne F, Luigi L, Etienne C, Guy B (1999) Differentiating lumbar disc protrusion, disc bulge, and discs with normal contours but abnormal signal intensity: magnetic resonance imaging with discographic correlations. Spine 24:44-53

21. Buirski G, Silberstein M (1993) The symptomatic lumbar disc in patients with low back pain: magnetic resonance imaging appearances in both a symptomatic and control population. Spine 18:1808-1811

22. Haughton V (1998) Spine imaging: should we be taking this lying down? Am J Neuroradiol 19:1594

23. Zamani AA, Moriarty T, Hsu L, Winalski CS, Schaffer JL, Isbister H, Schenck JF, Rohling KW, Jolesz F (1998) Functional MRI of the lumbar spine in erect position in the superconducting open-configuration MR system: preliminary results. JMRI 8:1329-1333

24. Jensen TS, Albert HB, Soerensen JS, Manniche C, Leboeuf-Yde C (2006) Natural course of disc morphology in patients with sciatica: an MRI study using a standardized qualitative classification system. Spine 31:1605-1612

25. Zou J, Yang H, Miyazaki M, Wei F, Hong SW, Yoon SH, Morishita Y, Wang JC (2008) Missed lumbar disc herniations diagnosed with kinetic magnetic resonance imaging. Spine 33:E140-E144 